An important proof of the writer's thesis is the fact that, under modern conditions, where often two and three generations have been schooled by mission or Chinese enterprise, the children requiring lenses reveal a marked diminishing of myopia and a relative increase of hypermetropia.

Diseases.-Briefly, trachoma is most general of the more malignant types, whole villages in North China being afflicted. Corneal ulcers, especially rodent, catarrhal and serpent, have left their scars and trail of blindness on innumerable eyes. Practitioners usually make a point of examining for cicatrices before other routine; hence the almost general use of keratomers. Next in severity are sarcoma, orbital tumours, various forms of retinitis and the almost endemic catarrhal and purulent conjunctivitis. Detachment of the retina is not so common as might be expected and cataract has no more than normal incidence except in midYangtze valley, where conditions approach those in parts of India. Ptosis, keratoconus, and other afflictions occur in more or less normal degree, but glaucoma is extremely rare. The first two causes, trachoma and ulcers, account for most of the 3 million blind in both eyes, and 5 million in one eye, as estimated a few years ago.

\title{
THE PRINCIPLES OF ORTHOPTIC TRAINING
} BY

\author{
Sheila Mayou
}

LONDON

Purpose.

It is well known that a patient who squints has monocular vision and it is our aim to give him binocular vision. It is realised that the squinter uses only one eye : what is happening to the vision of the other? It is obviously being suppressed unless there is diplopia present. The squint may be either an alternating strabismus, in which case the visual acuity of each eye would be equal, or it may be unilateral. A patient with a unilateral strabismus may or may not have an amblyopic eye. If there were no amblyopia present, it might be necessary partially to occlude the good eye, until the deviating eye was able to fix at will. If there were amblyopia present, the good eye would be either totally or partially occluded, until the patient was able to alternate.

The first stage is to teach the patient to obtain simultaneous perception, that is to say, to see an object with both eyes at the same time, and in order to do this, unless the visual axes are 
parallel, the patient must, at one time during his training, have diplopia. Once a patient has overcome amblyopia and suppression and has not sufficiently good binocular vision to overcome any deviation that may be present, he will have diplopia.

From simultaneous perception we shall pass to fusion, which is the mental power of blending the two images together, and thus convert the diplopia into binocular single vision.

The third and final grade is stereoscopic vision : the ability to judge distances and to appreciate depth.

Age.

The age of a patient is a very important question to anyone who is contemplating a cure by orthoptic exercises. In many cases the age depends on the type of case. Thus an esophoria of thirtyfive to forty years might receive a great deal of benefit from treatment, but in the case of a squint of the same age the exercises would probably overcome the suppression and the patient would be left with diplopia. By surgical means and further training, it would be possible in a young child to overcome the diplopia and obtain binocular vision, but where the squint is of long standing it is very doubtful. The diplopia once having been awakened might not easily be suppressed again. Again, where there is amblyopia of a high degree, it is not usually possible to recover the visual acuity once the patient is in his teens and therefore it would be rather a waste of time to attempt to cure the deviation by exercises. The most suitable age is, I think, between five and six years; should there be amblyopia present it is generally fairly easily overcome by total occlusion of the good eye. The child at this age should be sufficiently intelligent and interested to understand what is required of him. On the other hand I have had some children of two years old who have either attended for treatment or worked at home on small machines. These cases need endless patience and a great deal of help from the parents, otherwise it is better to leave the child until he is between three and four years of age, but the child should be kept constantly under supervision, to see that amblyopia does not develop.

Treatment has proved satisfactory with children up to the age of eighteen years, whether the exercises alone have been sufficient or whether the exercises with the aid of surgery have proved necessary. It is obvious that the younger the case, the greater will be the possibilities of obtaining a cure bv exercises only. A number of cases over the age of twenty where there has been a squint of long standing have developed binocular vision, but not always stereoscopic vision, and therefore it is inadvisable in cases of this type to encourage diplopia as it might not prove to be possible to suppress it again. 
Optical Correction.

Before attempting to test a patient's binocular vision, it is essential that any refractive error should be corrected, and that the patient should be accustomed to wearing glasses.

The optical correction plays an extremely important part in the treatment of convergent strabismus, and the cases which are most amenable to treatment are those where the angle of deviation is lessened when the proper optical correction is given.

The wearing of an optical correction is not sufficient, for if one eye were amblyopic, the eye would not function, and therefore the correction would be very little use to that eye, until it was made to function as well as the other. Amblyopia must first be overcome by the aid of an occluder and a proper optical correction. When the patient is able to use both his eyes to look at an object, he is then receiving the full benefit of his optical correction.

What is the use of prisms in a case of a squint? A prism is used to bring the retinal image to the deviating eye in the position required for fusion. This could only be effective if the deviating eye had an image perceived by the brain, that is to say, if diplopia was present. In the case of a child, it is very much better to encourage mental effort in overcoming the diplopia, if horizontal, but if vertical, treatment is often assisted by giving a small prism that will overcome half to three-quarters of the vertical imbalance. After the patient has obtained fusion with the correction, the prism may then be reduced and a further effort must be made to get binocular single vision, until the vertical imbalance has been entirely overcome and no prism is necessary.

A great number of patients are unable to read, and therefore do not know their letters or numbers, wherefore it becomes impracticable to use Snellen's Test Types for these young children. Vision is tested, however, either by making them tell us what toys they are able to see, or by means of a rotary "E." Several cards, with objects such as balls, dolls, etc., have been graded in a similar way to the Snellen's Test Types, so that it is possible to determine the visual acuity of each eye fairly accurately. The rotary "E," is a much more satisfactory and accurate test. The patient is given a metal " $\mathrm{E}$ " which is held in the hands, and in the distance he perceives a series of "E's," the one at the top being fairly large and the bottom ones quite small. They are graded in a similar way to the Snellen's Test Types. If the " $\mathrm{E}$ " on the card faces the left, then the patient places his also to look to the left; if the " $E$ " on the card faces downward, the patient must make his also look downward, and so on.

The visual acuity having been determined, it must then be decided whether it is necessary to occlude one eye. This depends on the age and if there is any amblyopia present. 
The occlusion may be carried out with a Doyne's disc, but during the past year I have been using adhesive plaster which has been stuck on the lens of the patient's glasses. The plaster is cut so that it overlaps the lens in all directions, and a special projection is made across the bridge of the nose.

The reason for this projection is to stop the child from looking through the corner of the patch. In the case of the disc, the child is apt, unless it is very well cut, to look through the side, but the

DIAGRAM I.

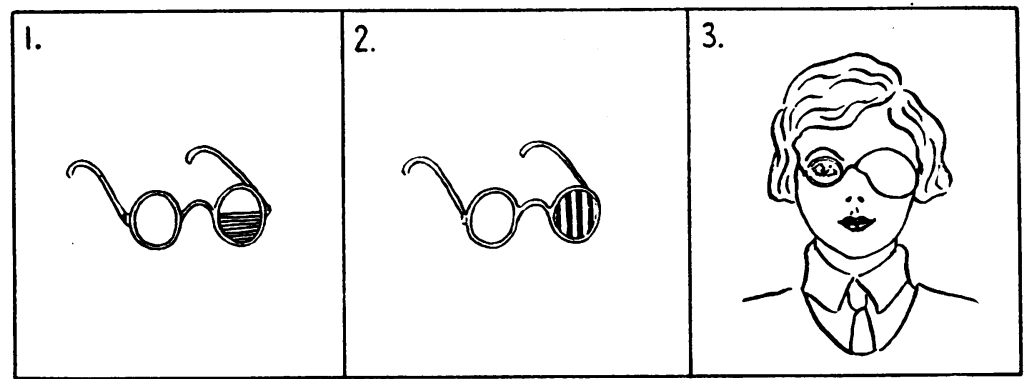

1. Partial occluder, the left eye will be totally occluded for reading only.

2. Partial occluder, the left eye will be partially occluded all the time.

3. Total occluder, the left eye will be totally occluded.

plaster being soft, moulds to the face and does not cause any irritation. (Diagram I, Fig. 3).

Where the visual acuity is $6 / 12$ a partial occluder can be used. This may take the form of strips down the centre of the lens of the good eye. Care should be taken to see that the child is using the amblyopic eye, as some patients prefer to look through the strips rather than use the non-fixing eye, in which case the good eye must be totally occluded. (Diagram I, Fig. 2).

Atropine may also be used as a means of recovering vision. Ointment or drops are placed every morning in the fixing eye. This is to encourage the patient to use the non-fixing eye for near work. Where there is amblyopia of $6 / 12$ or more, the child will continue to use the atropinised eye wherefore it is obvious that the use of atropine is not satisfactory in all cases.

Placing a plane glass over the atropinised good eye is another method of overcoming amblyopia. In many ways this is satisfactory, if the patient will use the amblyopic eye. Occlusion in some cases increases the angle of squint, whereas if the patient is having atropine he will still be able to get binocular vision for distant objects.

Another method of partial occlusion which acts in a similar way to atropine, is to paste a piece of paper on to the lower half of the 
lens in front of the fixing eye. For distant vision he will then be using both his eyes, as they will be looking through the upper portion of his glasses. For reading he will use the amblyopic eye only, through the lower half of his lenses. At the same time the lower half of the lens is used for the good eye. In this way distant binocular vision is obtained and the amblyopic eye is being used solely for near work. (Diagram I, Fig. 1).

Vision up to the age of twelve years in most cases will improve from $6 / 24$ to $6 / 6$, but above this age it is doubtful how much improvement can be expected. Cases with amblyopia are not usually taken on for treatment until the visual acuity of the amblyopic eye has reached $6 / 12$ or until the patient is able to alternate at will.

Having taken the visual acuity of each eye, both with and without glasses, before going any further, it is necessary to ascertain whether there is a weakness or paralysis, or limitation of any muscle.

The patient should also be tested for inconcomitancy. This is done roughly, by his first fixing the non-squinting eye and the angle of deviation is noted, subsequently the squinting eye is then fixed and the angle noted. If the convergence increases when fixing the squinting eye, the patient is said to be inconcomitant, and his prospects of a cure are dependent on whether the inconcomitancy is slight or very marked.

The patient is said to be concomitant if the angle of convergence appears to be the same when fixing either eye; also the degree of convergence must not increase when the patient looks to the nine points of the field of vision.

The patient is then tested on the Worth Four Lights test.

These lights test for binocular single vision in the distance, and consist of four lights, one red, two green and one white. The patient is given a red and a green glass, one in front of each eye.

If the patient has a squint he will see :

3 lights if he is suppressing one eye. The colours of the lights will depend on which eye is being suppressed.

3 or 2 lights if he alternates, first the red and then the green.

5 lights, in which case either the fusion is not sufficiently strong to overcome any small muscular defects, or the angle of deviation is too great to be overcome by fusion, and he may have diplopia.

4 lights, in which case he has binocular single vision.

If the squint is not of large degree, the deviation is usually measured by the Maddox Rod test and Wing test, using the rod 
DIAGRAM II.

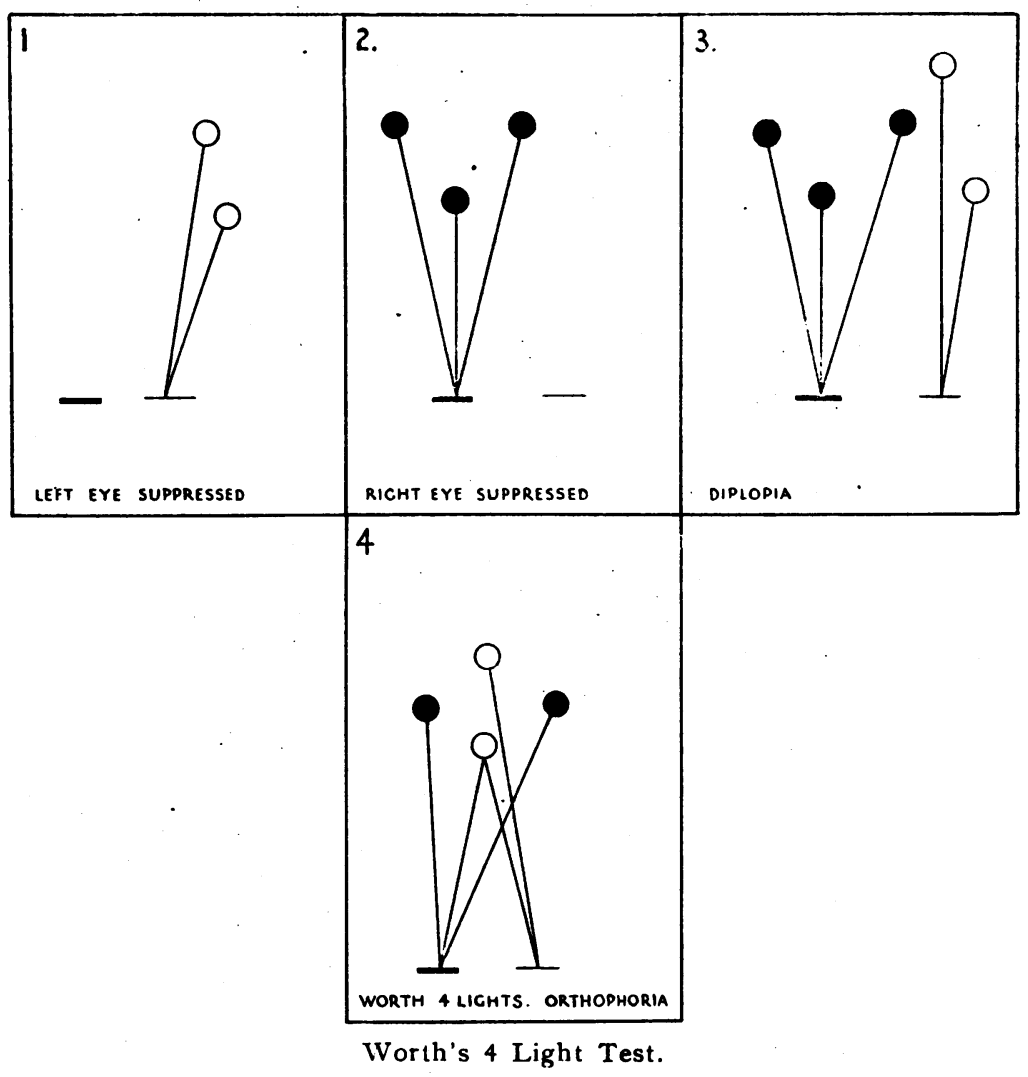

first in front of one eye and then in front of the other, in case there should be inconcomitancy.

The testing of binocular vision follows. The first stage, as has already been mentioned, is to obtain simultaneous perception, that is, to make the patient use both eyes together when looking at an object.

Suppression at this stage of treatment may fall into one of the three following groups :

1. Complete suppression.

2. Abnormal correspondence of retinal points or false projection (binocular).

3. Partial suppression.

The patient is seated in front of the synoptophore and given. two slides such as a lion and a cage, which have no fusion element 
present. He is asked to put the lion in the cage by moving the tubes. If he is unable to put the lion in the cage at any angle he is placed under the group "complete suppression." It is presumed that the area of suppression is so large that he is unable to perceive the images at the same time without alternating.

To overcome this suppression, the angle of squint is taken by noting the position of the corneal reflex in each eye when each is fixing separately, and then converging the tubes until the reflexes are in their original positions.

Noting the angle of deviation, the patient is told to fix the lion whilst the trainer passes the cage backwards and forwards over the region where he should see the lion in the cage. That is to say, if a case has a convergent strabismus of twenty degrees found by position of the corneal reflexes (this is known as an objective reading) then the cage would be moved between the angle of twenty-five and fifteen degrees, so that every time it passed backwards and forwards it would be passing over the macula of the non-fixing eye. In this way there is stimulation of the maculae of both eyes, since one is fixing the lion and the cage is being passed over the other. At first the two images will bear no relation to each other, but as the lesson continues the patient will notice that the cage appears to get nearer the lion. After a short while the cage will touch the lion shewing that the area of suppression is decreasing, eventually the cage gets over the lion and remains stationary, the lion is now " in the cage." On being asked to put the lion in the cage the patient will then place it in at the same angle as the trainer, that is to say if the angle has not lessened, at an angle of twenty degrees convergent, or if it has decreased, at a lower angle. He has then obtained simultaneous perception, since the objective reading (the angle obtained by the trainer by the position of the corneal reflexes) and the angle obtained by the patient, (the subjective reading) coincide. The next stage is to obtain simultaneous macular perception.

\section{Abnormal correspondence of retinal points or false projection.}

A number of patients fall under this group. For example, a child with good visual acuity in both eyes wearing glasses seems to have approximately twenty degrees of convergence which measured on the tangent scale proves to be true. The child is placed in front of the synoptophore, showing him a lion and a cage, he is asked to put the lion in the cage by moving the tubes. When he says that the lion is in the cage the degree of deviation is found to be five degrees of convergence only. On examining the child's eyes to see the position of the corneal reflexes it is noted that in one eye it is fairly central whereas in the other it is 
" on the outer margin of the cornea." If the child is asked to look from the centre of the lion to the centre bar of the cage, the eyes will alternate from one picture to the other. This is due to the fact that he is using the macula of one eye and some other point of the retina of the other, he looks first with the macula of one eye and then with the macula of the other eye.

Had he been using the maculae of both eyes, he would have put the lion in the cage at the actual angle of squint, namely twenty degrees and when asked to look from the centre of one picture to the centre of the other, there would have been no movement of either eye, since the two centres of the pictures coincide and the patient was using both maculae simultaneously.

The patient that puts the lion in the cage at a lower angle than the angle of deviation is said to have abnormal correspondence of retinal points or false projection. The angle at which the patient puts the two slides together is known as the subjective angle and the angle obtained by the trainer is the objective. The subjective and objective angles must coincide before attempting to develop binocular vision.

Having obtained the objective reading, which is approximately at a convergent angle of.twenty degrees, the patient is asked to fix the lion with his right eye, and the trainer passes the cage backwards and forwards over an angle of approximately twenty-five to twenty degrees.

Gradually the subjective angle (the angle at which the patient places the lion in the cage) will increase, and the objective angle will probably decrease until the two angles coincide, and the patient obtains simultaneous perception. Simultaneous macular perception must now be obtained.

It is interesting to note that the number of cases that have no binocular vision when first tested is fairly big, and this, I think, is one of the reasons why some squint operations have not turned out successfully, even though, after the operation was performed, the eyes stayed straight for a week or so.

Out of nine hundred cases which consisted of esophorias, exophorias, divergent and convergent squints, three hundred had no idea of binocular vision, that is to say one third of the total number.

These three hundred cases can be subdivided into those which had abnormal correspondence of retinal points, and those which had complete suppression when first tested.

Of three hundred cases, one hundred and forty-four had abnormal correspondence of retinal points, and one hundred and fifty-six had complete suppression. That is to say, of the three hundred cases who had no binocular vision, one half had 
abnormal correspondence and the other half had complete suppression. This, I think, points out the necessity for pre-operative treatment if a concrete result is the ultimate aim.

\section{Partial suppression.}

All cases who have just overcome suppression and non-correspondence of retinal points come under this heading. Cases which have some binocular vision but have an angle of deviation also fall in this class. The class may be subdivided into two groups which consist of :

a. Simultaneous macular perception.

$b$. Simultaneous foveal perception.

The cases that have just obtained normal correspondence of retinal points still have a large area of suppression to overcome; on

DIAGRAM III.

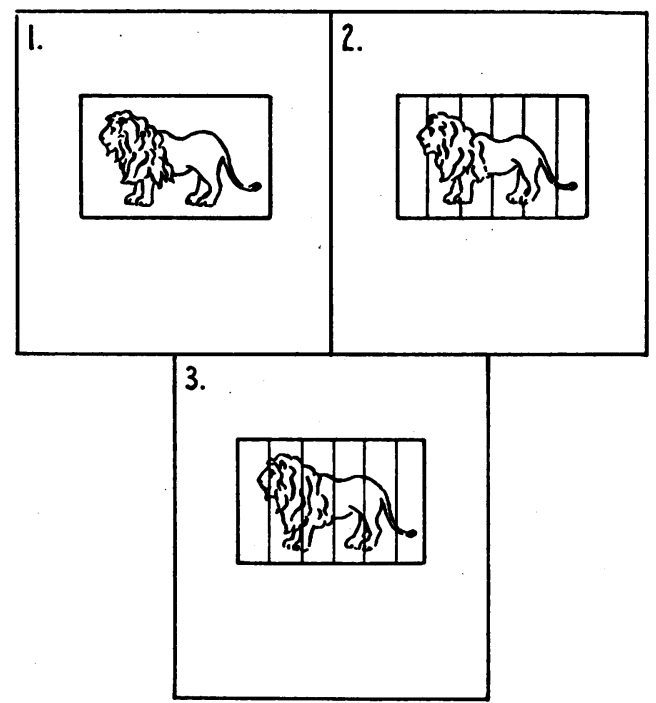

Diagram showing slides as seen by a patient with an area of suppression.

being asked what exactly they see, having put the lion in the cage. they will probably say that they see a lion with a square round him, but there are no bars at all. As the suppression decreases the bars of the cage will be seen at each end and then above and below the lion, but wherever the lion is there will be no bars. As the treatment continues the area of suppression will decrease; the patient will then be able to see all the bars, but the lion will appear to be in front of them, and then after a while he will be able to see 
the bars in front of the lion. The lion should then be placed in front of the other eye and the patient worked until he is able to see the bars of the cage in front of the lion, that is to say he has simultaneous macular perception. The slides used to determine this subtend an angle of eight degrees.

When the patient has obtained simultaneous macular perception we then try to obtain simultaneous foveal perception. To obtain this the patient is given a small image to look at, let us say a lion and cage, subtending a visual angle of three degrees, and in order to have simultaneous foveal perception the patient must see the bars of the cage in front of the lion. It is advisable not to attempt to teach a patient to fuse until he has simultaneous macular perception and has attempted to get simultaneous foveal perception. At this stage the patient should be encouraged to get diplopia. This is helped by the myoculator. The myoculator is a machine which improves ocular motility.

The myoculator moves an illuminated object across a wall. The pace at which the image is moved can be regulated, the range can be lengthened or shortened, and the direction can be changed.

The patient is first taught to alternate, by giving him red and green glasses and a septum. As the image travels across the screen, the patient is obliged to use first one eye and then the other. He will then notice that as he changes from one eye to the other, he will see two images simultaneously, one red and one green. The septum may now be removed and the patient should then be encouraged to see two images simultaneously, one red and one green. The red and green glasses should then be removed, and the patient will again suppress and see only one image, but after a while he will overcome this suppression and be able to see two images without the help of the red and green glasses.

\section{FUSION.}

Fusion is the mental power to blend into one the impressions received by the two eyes. In fusion exercises the patient is given two similar pictures which differ in some aspect from each other, but when blended together make a third picture which is different from the other two. For instance the following might be used (a) a bear with a red coat and black and white striped trousers, and (b) a bear with a white coat with black spots, and all blue trousers. When these two slides are fused, the resultant image should be one bear with a red coat and black spots, and blue trousers with black stripes. Where there is suppression, one image will disappear, so that it will not be possible to pass to the next stage, which is fusion with amplitude. (Diagram IV). 
DIAGRAM IV.

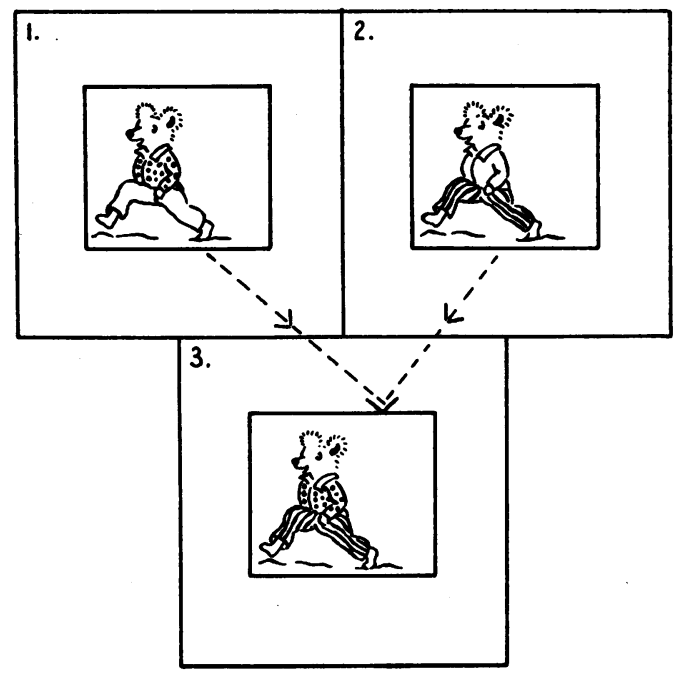

Diagram showing fusion slides.

Fusion ANd Amplitude.

This is the ability to maintain fusion while the pictures are abducted or adducted a few degrees. The normal abduction power on the synoptophore is eight to ten degrees and adduction power is forty degrees.

If there is suppression present the trainer may abduct or adduct the patient's eyes, but the patient will not notice when the fusion has broken. It is therefore necessary to be constantly watching the corneal reflexes of each eye to see that the patient is not being allowed to suppress.

The fusion angle is the smallest angle at which two slides can be fused together. That is to say when the slides are fused, and then either adducted or abducted until they part, the angle at which they rejoin is the angle of fusion.

Myoculator exercises.-Since the patient has fusion and some power of amplitude, and diplopia has been elicited, the patient must be taught to bring the two images together, so that they form one image seen by both eyes.

The patient is again given red and green glasses and asked to make a mental effort to get the two images seen on the screen into one. If the image is partly red and partly green, then it is obvious that he has binocular single vision, but should it be all red or all green it can be seen at once that he is suppressing and has only monocular vision instead of binocular. 
Stereoscopic vision.-Special slides for training perception of depth and teaching the patient to appreciate solidity have been designed for the training of stereoscopic vision on the synoptophore.

\section{TREaTMent and Machines.}

The treatment given to all patients for the main part is done on the synoptophore or synoptiscope, supplemented by the myoculator and various home work machines. It is possible on these machines to work a case with forty-five degrees of convergence, or forty degrees of divergence, and it is also possible to correct for fourteen dioptres of hyperphoria and thirty degrees of cyclophoria. A number of slides have been made for various stages of the treatment, in order to keep the patient's interest. These slides are in three groups and are used for the development of :

1. Simultaneous perception.

2. Fusion.

3. Stereoscopic vision.

The first group consists of two pictures which have nothing in common, there is no fusion element present, slides such as lions and cages, soldiers and boxes, spiders and webs, cars and garages etc., are used.

The second group for the development of fusion consists of two pictures exactly similar in every respect except that on each slide there is a small control mark. For instance, there might be two rabbits exactly similar, except that on one slide one rabbit would have no tail, but a bunch of flowers in his hand, and on the other he would have a tail but no flowers. When these two slides are superimposed the patient would see one rabbit with a tail and a bunch of flowers.

If the patient were suppressing, he would notice that he failed to see either the flowers or the tail, and therefore realise that the pictures were not properly joined. Other slides consisting of Mickey mouse, Pip, Squeak and Wilfred, and many others, are used so that the child may not get bored or tired with his exercises.

For the third stage, the development of stereoscopic vision, special slides have been designed so that the child is able to see whether one object in the picture appears nearer to him than the others. These exercises always prove to be amusing and are usually much appreciated by the patients.

In connection with the exercises on the synoptophore or synoptiscope the patients have home exercises, and these are carried out on small machines which are hired at a low rate from the 
opticians. The type of machine given to the patient depends on the stage the patient has reached in his training. For instance, it would be of no use to give a stereoscope with stereoscopic pictures to a child who is unable to fuse.

The home-work machines are graded for each stage in the treatment. For the development of simultaneous perception there is no homework machine. This stage must be achieved by a person who has been trained to give orthoptic exercises, as a parent or nurse could not possibly be expected to measure angle gammas or note positions of corneal reflexes.

After simultaneous perception has been developed, we can give a patient a machine to develop simultaneous macular perception and decrease the area of suppression. This machine may be one of two, either the Maddox Cheiroscope or the Strabismuscope.

On both these machines the patient is given sufficient prism help to correct the angle of deviation, and a special set of toys. These toys consist of butterflies and nets, babies in baths, counters and rings and soldiers in boxes, etc. The parent or the nurse is shown how to use them and instructed to make the child do the exercises at least twenty minutes every day.

For the development of fusion, fusion slides are given on the synoptophore and on the cheiroscope and strabismuscope, a special set of toys with a fusion element is given and worked in a similar way to the first set.

When fusion has been established, amplitude of fusion is developed. To do this, abduction exercises in the case of a convergent strabismus or esophoria, and adduction exercises in the case of a divergent strabismus or exophoria, are given on the synoptophore.

On the cheiroscope and strabismuscope the patient is given fusional drawings to strengthen the fusion. These instruments may now be replaced (if the fusion is sufficiently strong) or supplemented by a stereoscope in the case of a convergent strabismus or esophoria, and amblyoscope in the case of a divergent strabismus or exophoria.

In the case of a convergent strabismus or esophoria, the patient is given a set of fusion pictures and taught how to abduct his eyes by turning the rotary prism in the stereoscope whilst fusion is maintained. Since it is only possible to abduct about ten degrees, an amblyoscope would not be very beneficial in these cases.

Where there is a divergent strabismus or exophoria, the patient is given a set of fusion cards and an amblyoscope and is taught to adduct his eyes whilst fusion is maintained. Adduction after training is normally about forty degrees, and therefore a stereoscope with a rotary prism which will only accommodate twenty degrees will not be of very much use. 
For the development of stereoscopic vision, as on the synoptophore, where we have stereoscopic cards, Sattler's stereoscopic cards are used on the stereoscope, on the amblyoscope and strabismuscope a further set of stereoscopic cards are used which have been specially designed. Then, should the case prove to be rather long, there are alternative instruments that can be used at home, such as kinetic stereoscope, which can be used for increasing amplitude of fusion and improving ocular motility: also the diploscope, which has proved to be very useful in the final stages to teach the patient to control his eyes.

Considering now the question of surgical treatment of squint; the question arises as to when operation is indicated. Before orthoptic treatment started in the hospitals, the cases were generally operated on at between 14 and 21 years of age, if by this time they had not grown out of their squint. What was the result of the operation? 'The eyes were put straight, but since there was no binocular vision, there was nothing to keep them straight, and a great many of these cases returned for a further operation a few years later.

Nowadays, nearly all the non-paralytic squints are sent for training before their operation. Because such a high percentage of the cases that are seen have no idea of binocular vision, it is essential that all cases should, before their operation, have simultaneous macular perception, fusion and some power of amplitude. After operation it is only necessary to develop their stereoscopic vision.

If operation leaves the eyes slightly divergent or convergent, the fact that the patient has fusion gives him the desire for binocular vision, and he attempts to get the diplopia joined, so straightening the eyes. If there were no binocular vision he would suppress one image which would tend to increase the deviation, this might mean a further operation.

In short, orthoptic exercises should be given, whether an operation is necessary or not; exercises should be given before and after operation, in order to consolidate the surgeon's success.

The age at which the operation should be done, entirely depends on the patient. If the child is sufficiently intelligent and can concentrate on the exercises at five years of age, it can be seen whether the exercises alone will prove sufficient, or whether it will be necessary to aid the treatment by an operation, in which case the sooner it is performed the better.

Children who live in very poor districts and attend the hospital clinics often undergo an operation to hasten the cure, although in time orthoptic treatment would probably have effected it. The reason for this is that poor parents cannot possibly spare the time 
or money to bring their children to the clinic for treatment over a long period.

Before cases are operated on, degrees of deviation and hyperphoria and cyclophoria are carefully measured on the synoptophore, for the surgeon's guidance.

It is absolutely essential that child and parent should co-operate with the trainer. Occlusion must be carried out properly, otherwise the visual acuity will not improve. Home exercises must be done regularly, as one cannot expect to cure a patient who is only doing two sessions of forty minutes a week. The trainer must be persuasive and make the work interesting and at the same time must instil into the patient the desire for a cure. A patient must be made sufficiently interested to overcome small difficulties, or a satisfactory cure cannot be obtained.

\section{ABSTRACTS}

\section{I.--CONJUNCTIVA}

(1) Lyon, M. W. (Indiana).-Conjunctival myiasis. Due to the gadfly, oestrus ovis. Amer. Jl. Ophthal., June, 1935.

(1) Lyon reports a case of larvae from oestrus ovis deposited in the conjunctival sac. 'The larvae moved in a caterpillar manner by inserting the chitinous hooks in their anterior extremities into the conjunctival tissues. Their rate of progress was $2-3 \mathrm{~mm}$. per second. Nine were removed by cotton and one passed down the lacrymal duct but gave rise to no further trouble.

The author comments on the fact that the patient was able to localise accurately the precise site in the conjunctiva in which the larvae were active. The larvae are less than $1 \mathrm{~mm}$. long and can be seen with a binocular loupe x 10 magnification. Relief from irritation was obtained by using holocaine and epinephrine ointment.

\section{H. B. Stallard.}

(2) Tita (Cataniz).-A case of conjunctival infection by larvae of oestrus ovis. (Sul un caso di miasi congiuntivale; oestrus ovis). Rass. Ital. d'Ottal., September-October, 1935.

(2) In this case the infection was due to a goat which sneezed in the patient's face. This was followed immediately by great discomfort and sensation of a foreign body. A neighbour was able to wash out seven larvae; relief was immediate but the pain returned after a short time. 